\title{
Clinical Feasibility of a Free-Weight Strength-Training Program for Older Adults
}

\author{
Patricia A. Brill, PbD, Janice C. Probst, PbD, David L. Greenbouse, MD, \\ Bruce Schell, PbD, and Carol A. Macera, $P b D$
}

Background: An emerging trend in the field of gerontology is the recognition that older adults, especially the frail elderly population, can increase their levels of strength and thus improve functional capability. Social acceptance of physical frailty and provision of care and assistance to dependent persons has now turned to helping the frail elderly adults maintain or improve functional independence.

Methods: The purpose of our study was to show the feasibility and effectiveness of a low-cost strengthtraining program using free weights for increasing strength and functional fitness among older adult volunteers. Participants aged 73 to 94 years were residents of a multilevel care retirement community in Columbia, SC. The strength-training program, led by an instructor, used dumbbells and ankle weights and was conducted in a multipurpose recreation room at the retirement facility.

Results: Functional performance measures (timed chair stand, 6-meter walk, stair climb, balance) handgrip strength, and self-assessment of activity level were outcome measures. All 25 participants completed the strength-training program. The average program adherence rate of all participants was 87 percent. No participant injuries or other adverse effects were observed. Functional performance measures improved significantly among program participants, with the greatest improvement in the timed chair stand (33.5 percent improvement) and the stair climb (17.6 percent improvement).

Conclusion: Free-weight strength-training programs are appropriate for older adults, can be implemented in community settings, and are associated with significant improvement in functional performance. (J Am Board Fam Pract 1998;11:445-51.)

Musculoskeletal weakness has been cited as one of the most common causes of disability in the community, ${ }^{1}$ predisposing older adults to falls ${ }^{2-4}$ and limiting activities of daily living. Deconditioning, physical inactivity, or chronic disease results in decreased strength, power, endurance, flexibility, and range of motion, which in turn result in decreased ability to perform activities of daily living 5 and decreased functional performance. ${ }^{6}$ These consequences can have a profound effect on the ability of older adults to lead functional, independent lives. ${ }^{7,8}$

Functional fitness is the level of fitness neces-

Submitted, revised, 14 April 1998.

From the NGH/Marriott (PAB), Houston; and the Department of Family and Preventive Medicine, School of Medicine JCP, DLG, BS), and the Prevention Center, School of Public Health (CAM), University of South Carolina, Columbia. Address reprint requests to Patricia $\mathrm{A}$. Brill, $\mathrm{PhD}, 8550$ Katy Freeway, Suite 201, Houston, TX 77024-1864.

This work was partially supported by a grant from the University of South Carolina Research and Productive Scholarship Fund. Additional support was provided by the Prevention Center, School of Public Health, and the Department of Family and Preventive Medicine, School of Medicine, University of South Carolina, Columbia. sary for a person to take care of personal, household, social, and daily living needs as well as to maintain a residence at home. Improving the components of functional fitness (muscular strength, endurance, and power; flexibility; and range of motion), whether separately or in combination, can be important for maintaining such functional performance activities as climbing stairs, rising out of a chair, walking, or mobilizing a wheelchair. Hip and knee extensor strength, for example, is needed to stand from a sitting position. If the hip and knee flexors become tight and lose their flexibility, walking becomes limited, posture becomes stooped, activities require extra energy and fatigue occurs more quickly, balance is difficult to achieve, and gait will be affected. Similarly, shoulder flexors and elbow extensors assist with pushing up from a chair, a bed, or a bathtub, and deconditioning causes difficulty with these activities.

Research has shown that high-intensity strength training can have a profound effect on functional independence and quality of life in persons up to the age of 100 years. ${ }^{9}$ Strengthening upper and lower body muscles can help improve 
strength and endurance, bone density, flexibility, agility, balance, and gait, as well as increase the capacity to perform activities of daily living. Although increasing levels of strength might be the most effective health-enhancing action taken to improve the odds of continued independence, high-intensity, machine-based training is not generally available to all older adults. To reach at-risk older adults, low-cost interventions designed to provide effective prevention or rehabilitation need to be offered in a variety of settings.

Results of preliminary research by Brill et al $^{10}$ suggested that a strength-training program using free weights could improve upper and lower body strength (seated chest press and leg extension) as well as functional performance measures (6-meter walk, chair stand, stair climb, and balance) among elderly participants. The research reported here expands on the this earlier work by replicating the exercise-training program with a greater number of older adults. In addition, this project shows both the feasibility and the effectiveness of freeweight strength training for older adults in nonspecialized community settings.

\section{Methods}

\section{Study Design}

A pretest-posttest quasi-experimental design was used instead of the case-control design called for in the original research proposal. Case and control participants were to have been randomly assigned from among all volunteers for the training program, with case members receiving the training intervention during the first 8-week session, and the control group entering the program later. Potential participants refused random assignment, however, and insisted on full participation in the program from its inception. Because the project could not take place without cooperation of the host facility and its residents, a quasi-experimental design focusing on feasibility and effectiveness was used to continue the effort. The research team chose to proceed with a more limited design, recognizing that the absence of a control group weakens the generalizability of the study.

\section{Study Population}

The testing and training protocol was approved by the Research Review and Human Studies Subcommittee, University of South Carolina School of Medicine, Columbia. Participants were residents of the South Carolina Episcopal Home at Still Hopes, Columbia, an upper socioeconomic multilevel care facility that houses 212 residents who live independently (67 persons) or in assisted living (70), health care (44), or skilled dementia care (22) units. Persons in the health or skilled dementia care units were not eligible for participation. After the purpose of the study was presented at a resident meeting, 25 residents (18 percent) agreed to participate in the strength-training program.

The criteria for inclusion were residential status, age older than 65 years, and ambulatory (with or without an assistive device). Volunteers were to be excluded if they had a history of heart attack or stroke within the previous 6 months, unstable angina, or any condition that their physician believed might be worsened by exercise; no persons with potentially excluding conditions volunteered for participation. The purpose, potential benefits, possible risks, and procedures of the study were carefully explained. The 25 cognitively intact participants ( 24 women, 1 man) ranged in age from 73 to 94 years (mean $83.4 \pm 4.6$ years). Five participants (20 percent) used assistive devices ( 2 wheelchairs, 2 walkers, and 1 cane). Written informed consent was obtained from all participants.

\section{Testing Protocols}

All participants underwent baseline handgrip strength, balance, and functional performance examinations the week before the training program began. These assessments, administered by the principal investigator, have been used by other researchers to measure strength and functional performance.

Using a self-assessment tool validated in a previous study, ${ }^{10}$ participants rated their level of physical (walking, exercise class, aerobics, swimming), household (laundry, making beds, dusting, sweeping), and social (getting out to attend functions or parties and visiting friends) activity before starting the strength-training program. Level of activity was rated on the following 4-point scale: 1 - very active, 2 - somewhat active, 3 - occasionally active, and 4 - not at all active. Scores from the three activity categories were summed to derive the summary activity score.

\section{Handgrip Strength}

Handgrip strength, using the squeezing muscles of the dominant and nondominant hand, was 
measured as an index of static grip strength needed for such activities as rising from a chair or bathtub; this measurement also determined the initial dumbbell weight for each resident. Handgrip was measured by a Smedeley II handgrip dynamometer. Three trials were given, alternating the right and left hand. Scores were recorded to the nearest kilogram, and the best score for the right and left hand was used for analyses.

\section{Physical Function}

Leg strength, power, and functional mobility were measured by a timed chair stand, a 6-meter walk, the number of steps required to walk 6 meters, and a stair-climb test. For the chair-stand test, participants were instructed to cross their arms over their chest, and on command of "go," stand up as fast as possible. Their standing time was measured by a stopwatch to the nearest 0.01 second from the start command until a balanced, upright stance was achieved.

Two trials were given, with the fastest time used for analyses. Gait velocity was measured to the nearest 0.01 second as participants traveled a distance of 6 meters. The participants started behind a line on the floor and walked as fast as they could across a second line 6 meters apart. The fastest of two trials and the number of steps taken to walk the 6 meters were recorded. Participants were not allowed to use any assistive devices to complete the 6-meter walk.

During the stair-climb test participants were timed to the nearest 0.01 second while climbing up one flight of stairs (with banisters) (one trial) from the command "go" until both feet reached the first landing (seven steps).

\section{Balance}

Balance was measured by having the participants assume the following three different standing positions for a maximum of 10 seconds: parallel (feet together), semi-tandem (feet together with toes next to instep), and tandem (heel to toe). The combined total number of seconds participants were able to hold all three positions was recorded for a score of 0 to 30 .

\section{Physical Performance Summary Measure}

A summary measure of physical performance was computed based upon four separate tests of functional performance using the method outlined by
Seeman et al. ${ }^{11}$ The four functional performance measures included timed measures of balance, gait, chair stand, and stair climb. Scores for each of the functional performance measures were rescaled to a range of 0 to 1 before summing them, with 1 indicating the best performance and 0 , the worst. Rescaling was accomplished by dividing a participant's raw score by the maximum value from all of the participants, creating a value analogous to a percentage, with a range of 0 to 1 . For all tests except balance, higher raw scores indicated worse performance. For these subscales, results of the division were subtracted from 1 to reverse the order of scores, so that the higher scores would reflect better performance. The rescaled subscale scores thus ranged from 0 (worst) to 1 (best) and represented the proportion of the best score that the participant achieved. All assessments were conducted by the principal investigator.

\section{Intervention Program}

After the 25 residents completed all baseline strength, balance, and functional performance tests, they entered into the training program. Exercise sessions were conducted by a member of the University of South Carolina coaching staff who had an undergraduate degree. This person was trained and supervised by the principal investigator, who attended all of the exercise sessions, ensuring each participant used correct form while performing each exercise. The strength-training sessions, which lasted 30 minutes three times a week, were conducted in the main activity room of the facility. The only special items obtained for the strength-training program were dumbbells and ankle weights, which were placed on a utility cart and stored in a closet between sessions. Participation at each session was accounted for by the participants signing a log sheet.

Initial dumbbell weight for the training group was determined by taking 10 percent of the participant's dominant handgrip score. Beginning dumbbell weights ranged from 1 to 4 pounds. Because of severe disabling arthritis or other medical conditions (eg, stroke, mastectomy), a few participants were not able to lift the same weight in both hands. These participants were given different weights for the right and left hand. All participants began with 1-pound ankle weights.

Each exercise session consisted of performing five upper body (alternate military press, front 
Table 1. Pretest and Posttest Means, Mean Differences, and Percentage of Change in Functional Performance Variables.

\begin{tabular}{lrrrrrr}
\hline Functional Performance & \multicolumn{2}{c}{ Pretest } & \multicolumn{2}{c}{ Posttest $^{2}$} & $\begin{array}{c}\text { Mean } \\
\text { Difference }\end{array}$ & $\begin{array}{c}\text { Percent } \\
\text { Improvement }\end{array}$ \\
\hline Right hand grip (kg) & 19.5 & 6.2 & 19.7 & 5.8 & 0.28 & 1.4 \\
Left hand grip (kg) $^{\star}$ & 18.2 & 5.9 & 19.6 & 6.2 & 1.28 & 7.0 \\
Gait, 6-meter steps (n) $^{*}$ & 11.4 & 3.9 & 11.0 & 2.9 & -0.45 & 3.9 \\
Gait, 6-meter walk (sec) $^{\star}$ & 6.0 & 3.2 & 5.1 & 2.3 & -0.80 & 13.3 \\
Chair stand (sec) $^{*}$ & 2.0 & 2.0 & 1.4 & 1.0 & -0.67 & 33.5 \\
Stair climb (sec) $^{\dagger}$ & 9.0 & 11.3 & 6.1 & 6.4 & -1.59 & 17.6 \\
Balance (sec) $^{\dagger}$ & 23.0 & 5.6 & 24.8 & 5.7 & 1.86 & 8.1 \\
Summary performance $^{\ddagger}$ & 3.1 & 0.7 & 3.3 & .5 & 0.20 & 6.4 \\
\hline
\end{tabular}

Note: Higher scores reflect better performance.

$\mathrm{SD}$ - standard deviation.

*Significant difference $(\mathrm{P}<0.01)$ between pretest and posttest means.

+Significant difference $(\mathrm{P}<0.05)$ between pretest and posttest means.

¥Significant difference $(\mathrm{P}<0.001)$ between pretest and posttest means.

Summary performance score based upon times from the 6-meter walk, stair climb, chair stand, and balance tests.

shoulder raise, lateral raise, biceps curl, triceps extension) and five lower body (squats, knee raise, leg curl, toe raise, leg kick to side) strength exercises using dumbbells and ankle weights. The exercises were selected to improve the various activities of daily living, such as walking, transferring, stair climbing, bathing, dressing, lifting, and lowering, The training load increased progressively. Two sessions of 8,10 , then 12 repetitions of cadence exercises were performed during a 2 -week period. At the beginning of each 2-week period, dumbbell and ankle weights were increased by 1 pound for all participants, and the number of repetitions at the new weight level began again at 8 . Approximately 95 percent of the participants progressed in weight every 2 weeks.

We did not reassess strength every 2 weeks, as researchers using large strength-training equipment had done. It was our intent to design a strength-training program that could be conducted within a residential facility with minimal effort. All participants were encouraged to increase their weight every 2 weeks. Participants who started the program using 1-pound weights finished the program using 5-pound weights. A 10-minute warm-up and cool-down period accompanied the strength-training session.

\section{Posttests}

Upon completion of the 8-week training program, all tests performed during the baseline evaluation were readministered to determine whether changes in handgrip strength, balance, and functional performance measures had occurred.

\section{Statistical Analysis}

The SAS statistical package ${ }^{12}$ was used for data analysis. Statistical significance $(P<0.05)$ was determined by using a general linear model (PROC GLM). Repeated measures analysis of covariance methods were used to determine whether significant differences occurred in strength and functional performance measures as a result of participation in the strength-training program.

\section{Results}

All 25 participants completed the strength-training program. The average adherence rate of all of the participants to the program was 87 percent.

Means and mean differences in activity, strength, and functional performance variables assessed before and after training are displayed in Table 1. Left handgrip and functional performance variables improved significantly. The time required to complete the chair stand, stair climb, and 6-meter walk decreased significantly, whereas the time participants were able to retain balance increased significantly. The greatest changes in performance occurred with the timed chair stand, stair climb, and 6-meter walk, for a 33.5 percent, 17.6 percent, and 13.3 percent improvement, respectively.

All self-reported activity levels were higher subsequent to the training program (Table 2). 
Table 2. Pretest and Posttest Means, Mean Differences, and Percentage of Change in Self-Reported Activity.

\begin{tabular}{lcccccc}
\hline \multirow{2}{*}{ Activity } & \multicolumn{2}{c}{ Pretest } & \multicolumn{2}{c}{ Posttest } & \multicolumn{2}{c}{ Mean } \\
Dean & SD & Mean & SD & Pifference & Improvement \\
\hline Physical & 2.0 & 0.7 & 1.7 & 0.5 & -0.25 & 12.5 \\
Household & 2.1 & 0.9 & 1.9 & 0.9 & -0.13 & 6.2 \\
Social & 1.8 & 0.9 & 1.6 & 0.6 & -0.21 & 11.7 \\
Summary & 6.0 & 1.8 & 5.2 & 1.3 & -0.75 & 12.5 \\
\hline
\end{tabular}

Note: Lower score indicates higher activity level.

SD - standard deviation.

"Summary activity score based upon total added score of physical, household, and social activities.

†Significant difference $(\mathrm{P}<0.05)$ between pretest and posttest means.

Self-reported physical activity also increased significantly. Increases in self-reported household and social activities, while not statistically significant, are in the predicted direction. When all activities are included in a summary scale, overall activities increased significantly.

\section{Discussion}

Numerous studies now document the ability of older, frail adults to improve their level of strength through participation in a moderate- to high-intensity strength-training program. ${ }^{9,13-20} \mathrm{High}$-intensity strength-training on large strength-training equipment (greater than 80 percent 1 repetition maximum) has been reported to improve functional status, increase activity levels, counteract weakness, and decrease physical frailty in older adults. ${ }^{9,11,14}$ Consistent with the findings of other 8-week studies, $9,13,15,20$ our elderly participants showed significant improvements in functional performance and modest improvements in self-reported physical activities subsequent to an 8-week exercise period. These findings suggest that in an elderly population a community-based, low-cost strength-training intervention using free weights can bring about improvements in functional performance comparable to those gained from using large strength-training equipment., ${ }^{9}, 14$

The free-weight strength-training program was well received by the elderly residents of Still Hopes. Of the 100 residents, 25 volunteered to participate. All volunteers remained with the program throughout the study, and overall attendance at sessions was high (87 percent). No injuries occurred as a result of the training sessions. In response to the participants' request, a video of the exercise session was produced and left at the facility after the study concluded, so the residents could continue with the program on their own. Overall, the subjective impression was that the residents enjoyed the exercise program, took pride in participating in it, and found it enhanced their self-esteem. Furthermore, the program was sufficiently in demand that the facility institutionalized the exercise program after the grant period ended.

A free-weight strength-training program has modest equipment requirements and can be instituted in many facilities serving older adults. Total equipment costs (dumbbells and ankle weights) for this project, which could accommodate up to 15 persons per session, were $\$ 600$. Donations of weight equipment or raising money through bake sales or arts and crafts sales could help offset the costs of the equipment. In addition, facilities housing a physical therapy department might already have some of the equipment needed to conduct a strength-training program for its residents.

The instructor, who led the exercises and supervised the 25 participants during each session, was paid $\$ 15$ per hour. An activity director, rehabilitation aide, or volunteer could be taught to conduct the strength-training program correctly and safely, eliminating the cost of an outside instructor. Motivational skills are more important than previous physical education experience. Chairs for participant seating were already available in the facility's multipurpose room, and the equipment was housed in an adjacent closet. After proper instruction on how to perform each exercise correctly, minimal supervision was necessary to ensure the safety of the participants.

In contrast, the cost for isokinetic or isotonic strength-training equipment used in previous studies to train similar muscle groups ranges from $\$ 7,500$ to $\$ 24,000$. Furthermore, when large strength-training equipment is used by elderly 
persons, who might have impaired vision, hearing, or mobility, close participant supervision is required. An instructor could safely supervise two participants at a time. It is unlikely that most institutions serving older adults, such as community centers or retirement facilities, could afford the space, equipment, and personnel required to maintain a training program using large strengthtraining equipment.

A limitation of this study is that neither study participants nor persons conducting the strength and functional performance assessments were blinded to the purposes of the study. For budgetary reasons, pretraining and posttraining measurements were conducted by the principal investigator. The objective nature of the assessments should have served to reduce researcher bias, but the possibility cannot be excluded. In addition, participants might have worked harder during their posttraining assessments to please the research team.

A second limitation is posed by the high functioning levels of most participants, which restricted the range of improvement along the measurements used. Exploration of the feasibility and effectiveness of free-weight training among more diverse populations is needed. Finally, the present study, which took only 2 months, did not address the issue of maximum functional performance gains: how much can function be improved in older adults, and how long does it take to reach the maximum? Similarly, time limitations prohibited the study from addressing the issue of retention of functional performance. Additional research is needed to document long-term effects on health and well-being.

This study shows that a strength-training program for older adults, using supervised exercises with dumbbells and ankle weights, can be implemented successfully in a community setting. Although further research is needed to determine any long-term benefits derived from free-weight exercise programs, wider use of such low-cost interventions could make it possible for older adults to regain functional abilities that have been lost through physical inactivity, maintain functional independence for a longer time, and require lower levels of assistance after injury or disease has impaired functioning. Finally, the major contribution of this investigation stems from its ability to assess the clinical feasibility of a simple, inexpen- sive strength-training program using free weights. Family physicians should be knowledgeable in the benefits of strength training for older adults and recommend such programs for their patients.

We would like to acknowledge Dana Flemming for assistance with the assessments, Doug Beavers for conducting the strengthtraining program, Mark Leski, $\mathrm{MD}$, for consultation on the functional fitness strength-training program, Dorothy Davis for data management and statistical analyses, Kirby Jackson for statistical consultation, and Pat Freund for her assistance in obtaining the equipment.

\section{References}

1. Levit KR, Freeland MS, Waldo DR. National health care spending trends: 1988. Health Aff Millwood 1990;9:174-84.

2. Cwikel J, Fried AV. The social epidemiology of falls among community-dwelling elderly: guidelines for prevention. Disabil Rehabil 1992;14:113-21.

3. Lord SR, McLean D, Stathers G. Physiological factors associated with injurious falls in older people living in the community. Gerontology 1992;38:33846.

4. Whipple RH, Wolfson LI, Amerman PM. The relationship of knee and ankle weakness to falls in nursing home residents: an isokinetic study. J Am Geriatric Soc 1987;35:13-20.

5. Hyatt RH, Whitelow MN, Bhat A, Scott S, Maxwell JD. Association of muscle strength with functional status of elderly people. Age-Ageing 1990;19:330-6.

6. Bassey EJ, Fiatarone MA, O'Neill EF, Kelly M, Evans WJ, Lipsitz LA. Leg extensor power and functional performance in very old men and women. Clin Sci Colch 1992;82:321-7.

7. Pendergast DR, Calkins EC, Fisher NM, Vickers R. Muscle rehabilitation in nursing home residents with cognitive impairment: a pilot study. Am J Alzh Care Rel Dis Res 1987; July/Aug:20-5.

8. Thompson RF, Crist DM, Marsh M, Rosenthal M. Effects of physical exercise for elderly patients with physical impairments. J Am Geriatr Soc 1988;36: 130-5.

9. Fiatarone MA, Marks EC, Ryan ND, Meredith CN, Lipsitz LA, Evans WJ. High-intensity strength training in nonagenarians. Effects on skeletal muscle. JAMA 1990;263:3029-34.

10. Brill PA, Jensen RL, Koltyn KF, Morrow J, Jackson AW, Keller MJ, et al. The feasibility of conducting a group-based progressive strength training program in residents of a multi-level care facility. Activities Adaptation Aging 1998;22:53-63.

11. Seeman TE, Charpentier PA, Berkman LF, Tinetti ME, Guralnik JM, Albert $M$, et al. Predicting changes in physical performance in a high-functioning elderly cohort: MacArthur studies of successful aging. J Gerontol 1994;49:M97-M108. 
12. SAS statistical program, version 6 [computer program]. Cary, NC: SAS Institute, 1996.

13. Connelly DM, Vandervoort AA. Improvement in knee extensor strength of institutionalized elderly women after exercise with ankle weights. Physiother Can 1995;47,15-23.

14. Fiatarone MA, O'Neill EF, Ryan ND, Clements KM, Solares GR, Nelson ME, et al. Exercise training and nutritional supplementation for physical frailty in very elderly people. N Engl J Med 1994; 330:1769-75.

15. Fisher NM, Pendergast DR, Calkins E. Muscle rehabilitation in impaired elderly nursing home residents. Arch Phys Med Rehabil 1991;72,181-5.

16. Jones CJ, Rikli RE, Benedict J, Williams P. Effects of a resistance training program on leg strength and muscular endurance of older women. JAPA 1994; 2:182-95.

17. McMurdo ME, Rennie LM. A controlled trial of exercise by residents of old people's homes. Age-Ageing 1993;22:11-5.

18. McMurdo ME, Rennie LM. Improvements in quadriceps strength with regular seated exercise in the institutionalized elderly. Arch Phys Med Rehabil 1994; 75:600-3.

19. Nichols JF, Hitzelberger LM, Sherman JG, Patterson P. Effects of resistance training on muscular strength and functional abilities of communitydwelling older adults. JAPA 1995;3:238-50.

20. Mihalko SL, McAuley E. Strength training effects on subjective well-being and physical function in the elderly. JAPA 1996;4:56-68. 